\title{
ON ABSTRACT HOMOMORPHISMS OF SOME SPECIAL UNITARY GROUPS
}

\author{
IGOR A. RAPINCHUK AND JOSHUA RUITER
}

\begin{abstract}
We analyze the abstract representations of the groups of rational points of even-dimensional quasi-split special unitary groups associated with quadratic field extensions. We show that, under certain assumptions, such representations have a standard description, as predicted by a conjecture of Borel and Tits [5]. Our method extends the approach introduced by the first author in [16] to study abstract representations of Chevalley groups and is based on the construction and analysis of a certain algebraic ring associated to a given abstract representation.
\end{abstract}

\section{INTRODUCTION}

The goal of this paper is to analyze the abstract representations of the groups of rational points of even-dimensional quasi-split special unitary groups associated with quadratic field extensions. Our main result, whose precise formulation is given in Theorem 1.2 below, is that, under certain assumptions, such representations have the expected description, as predicted by the following longstanding conjecture of Borel and Tits ([5, 8.19]).

As usual, for an algebraic group $G$ defined over a field $k$, we denote by $G^{+}$the (normal) subgroup of $G(k)$ generated by the the $k$-points of the unipotent radicals of the $k$-defined parabolic subgroups of $G$. With these notations, Borel and Tits conjectured the following.

Let $G$ and $G^{\prime}$ be algebraic groups defined over infinite fields $k$ and $k^{\prime}$, respectively. If $\rho: G(k) \rightarrow G^{\prime}\left(k^{\prime}\right)$ is any abstract homomorphism such that $\rho\left(G^{+}\right)$is Zariski-dense in $G^{\prime}\left(k^{\prime}\right)$, then there exists a commutative finite-dimensional $k^{\prime}$-algebra $A$ and a ring homomorphism $f_{A}: k \rightarrow A$ such that $\rho=\sigma \circ r_{A / k^{\prime}} \circ F$, where $F: G(k) \rightarrow G_{A}(A)$ is induced by $f_{A}\left(G_{A}\right.$ is the group obtained by change of scalars), $r_{A / k^{\prime}}: G_{A}(A) \rightarrow R_{A / k^{\prime}}\left(G_{A}\right)\left(k^{\prime}\right)$ is the canonical isomorphism (here $R_{A / k^{\prime}}$ denotes the functor of restriction of scalars), and $\sigma$ is a rational $k^{\prime}$-morphism of $R_{A / k^{\prime}}\left(G_{A}\right)$ to $G^{\prime}$.

If an abstract homomorphism $\rho: G(k) \rightarrow G^{\prime}\left(k^{\prime}\right)$ admits a factorization as in (BT), we will say that $\rho$ has a standard description.

Remark 1.1. It has been pointed out by B. Conrad and G. Prasad that by using the results of [7, Chapter 9] (particularly Proposition 9.9.2), one can construct counterexamples to (BT) over all local and global function fields of characteristic 2 (or, more generally, over any field $k$ of characteristic 2 such that $\left.\left[k: k^{2}\right]=2\right)$. The groups that arise in these counterexamples are perfect and $k$-simple. So, one should exclude fields of characteristic 2 in the statement of (BT).

In [16], the first author introduced a method, based on the construction and analysis of certain algebraic rings, for studying abstract representations of the elementary subgroups of simply-connected Chevalley groups over commutative rings. Using these techniques, he obtained a general result on abstract representations that, in particular, yielded (BT) in the case where $k$ is a field of characteristic $\neq 2$ or $3, k^{\prime}=K$ is an algebraically closed field of characteristic 0 , and $G$ is a split simply-connected $k$ group. Subsequently, this approach was extended in [17] to confirm (BT) for abstract representations of groups of the form $\mathbf{S L}_{n, D}$, where $D$ is a finite-dimensional central division algebra over a field of characteristic zero. In positive characteristic, results on this problem were obtained by Seitz in [20], which were subsequently revisited by Boyarchenko and the first author in [6] from the point of

2010 Mathematics subject classification: primary 20G15, secondary 20 G35.

Key words and phrases. Abstract homomorphism, special unitary group. 
view of algebraic rings. Furthermore, the first author recently employed the methods of [16], together with some considerations on central extensions, to obtain the first unconditional rigidity statements for finitely generated linear groups other than arithmetic groups/lattices ( $\operatorname{such}$ as $\mathrm{SL}_{n}(\mathbb{Z}[x])$, for $n \geq 3$ ) cf. [19]. We refer the reader to [18] for a more extensive overview of work on (BT) and its connections to various classical forms of rigidity.

In the present paper, we consider the following situation. Let $k$ be a field of characteristic 0 and let $L=k(\sqrt{d})$ be a quadratic extension. We set $\tau: L \rightarrow L$ to be the nontrivial element of $\operatorname{Gal}(L / k)$ and note that for any commutative $k$-algebra $R$, the action of $\tau$ naturally extends to $R_{L}:=R \otimes_{k} L$ via the second factor. For ease of notation, we will write $\tau(x)=\bar{x}$ for $x \in R_{L}$. Next, fix an integer $n \geq 2$ and let $V=L^{2 n}$ be a $2 n$-dimensional $L$-vector space equipped with a (skew-)hermitian form $h: V \times V \rightarrow L$. We will assume that $h$ has maximal Witt index, so that, with respect to a suitable basis of $V$, the matrix of $h$ is

$$
H=\left(\begin{array}{ccccc}
0 & -1 & & & \\
1 & 0 & & & \\
& & \ddots & & \\
& & & 0 & -1 \\
& & & 1 & 0
\end{array}\right)
$$

Let $G=\mathrm{SU}_{2 n}(L, h)$ be the corresponding special unitary group. Explicitly, for a commutative $k$ algebra $R$, we have

$$
G(R)=\left\{X \in \mathrm{SL}_{2 n}\left(R_{L}\right) \mid X^{*} H X=H\right\}
$$

where for $X=\left(a_{i j}\right)$, we let $X^{*}=\left(\bar{a}_{j i}\right)$ denote the conjugate transpose matrix. It is well-known that $G$ is a quasi-split simply-connected $k$-group (in fact, a $k$-form of $\mathrm{SL}_{2 n}$ ) whose relative root system $\Phi_{k}$ is of type $\mathrm{C}_{n}$ (see, for example, [2, Ch. V, §23.8] or [15, Ch. 2, §2.3.3]). With these notations, our main result is as follows.

Theorem 1.2. Let $L=k(\sqrt{d})$ be a quadratic extension of a field $k$ of characteristic 0 , and for $n \geq 2$, set $G=\mathrm{SU}_{2 n}(L, h)$ to be the special unitary group of a (skew-)hermitian form $h: L^{2 n} \times L^{2 n} \rightarrow L$ of maximal Witt index. Let $K$ be an algebraically closed field of characteristic 0 and consider an abstract representation

$$
\rho: G(k) \rightarrow \mathrm{GL}_{m}(K)
$$

Set $H=\overline{\rho(G(k))}$ to be the Zariski closure of the image of $\rho$ (we note that $H$ is automatically connected - see Lemma 4.1). Then if the unipotent radical $U=R_{u}(H)$ of $H$ is commutative, there exists a commutative finite-dimensional $K$-algebra $A$, a ring homomorphism $f: k \rightarrow A$ with Zariski-dense image, and a morphism of algebraic K-groups $\sigma: G(A) \rightarrow H$ such that $\rho=\sigma \circ F$, where $F: G(k) \rightarrow$ $G(A)$ is the group homomorphism induced by $f$.

(In this statement, we view $G(A)$ as an algebraic $K$-group using the functor of restriction of scalars — see $\$ 4$ for further details.)

The proof of this result proceeds along the following lines. First, since $G$ is a simply-connected $k$-group whose relative root system $\Phi_{k}$ is of type $C_{n}$, it follows that $G$ contains a $k$-split simplyconnected $k$-group $G_{0}=\mathrm{Sp}_{2 n}$ of type $\mathrm{C}_{n}$ (see [3, Théorème 7.2] or [7, Theorem C.2.30]). We consider the restriction of $\rho$ to $G_{0}(k)$, and use the construction given in [16] to associate to $\left.\rho\right|_{G_{0}(k)}$ an algebraic ring $A$, together with a ring homomorphism $f: k \rightarrow A$ with Zariski-dense image. Since $k$ and $K$ are both fields of characteristic $0, A$ is in fact a finite-dimensional $K$-algebra (by [16, Lemma 2.13(ii), Proposition 2.14]). As shown in [16], the algebraic ring $A$ plays a central role in proving that $\left.\rho\right|_{G_{0}(k)}$ has a standard description; it turns out that $A$ also suffices for the analysis of $\rho$. More precisely, following the general strategy of [16] and [17], we first show that $\rho$ lifts to a representation $\widetilde{\sigma}: \widetilde{G}(A) \rightarrow \mathrm{GL}_{m}(K)$, where $\widetilde{G}(A)$ is the generalized Steinberg group introduced by Stavrova [22] (which builds on an earlier construction due to Deodhar [8]). Then, using the fact that the kernel of the canonical map $\widetilde{G}(A) \rightarrow G(A)$ is central (which extends a result of Stavrova to the present situation), together with our assumption that the unipotent radical $R_{u}(H)$ is commutative, we establish the existence of the required algebraic representation $\sigma: G(A) \rightarrow \mathrm{GL}_{m}(K)$. 
The structure of the paper is as follows. We begin by recalling in several key definitions and statements pertaining to elementary subgroups of isotropic reductive group schemes (defined by Petrov and Stavrova [13]) that are needed for our purposes. We also discuss some relevant aspects of Stavrova's generalization in [22] of the classical theory of Steinberg groups and central extensions. Next, in $\$ 3$, we introduce the algebraic ring $A$ and, after establishing some preliminary statements, show that $\rho$ lifts to a representation $\widetilde{\sigma}$ of $\widetilde{G}(A)$. Finally, in $\widetilde{4}$, we follow the general strategy of [16] to prove that $\widetilde{\sigma}$ descends to a rational representation $\sigma$ of $G(A)$.

Notations And COnventions: All rings will be assumed to be unital and commutative. Unless stated otherwise, we will denote by $G$ the special unitary group $\mathrm{SU}_{2 n}(L, h)$ introduced above.

\section{Elementary subgroups And Steinberg Groups}

In this section, we recall several aspects of the theory of elementary subgroups of isotropic reductive group schemes, developed by Petrov and Stavrova [13], as well as relevant points of Stavrova's [22] generalization of the classical Steinberg group. In view of our later applications, we will focus on the case of $\mathrm{SU}_{2 n}(L, h)(n \geq 2)$.

2.1. Elementary subgroups. Suppose $\mathcal{G}$ is a reductive group scheme over a ring $R$ that is isotropic of rank $\geq 1$ (i.e. every semisimple normal $R$-subgroup of $\mathcal{G}$ contains a 1-dimensional split $R$-torus). Then $\mathcal{G}$ contains a pair of opposite parabolic $R$-subgroups $P$ and $P^{-}$that intersect properly every semisimple normal $R$-subgroup of $\mathcal{G}$ (such subgroups are sometimes referred to as strictly parabolic). In [13, the corresponding elementary subgroup $E_{P}(R)$ is then defined as the subgroup of $\mathcal{G}(R)$ generated by $U_{P}(R)$ and $U_{P-}(R)$, where $U_{P}$ and $U_{P^{-}}$are the unipotent radicals of $P$ and $P^{-}$, respectively (we note that when $R=k$ is a field, then $E_{P}(k)$ coincides with the group $\mathcal{G}^{+}$appearing in the statement of $(\mathrm{BT})$ ). The main result of [13] (see also [21, Theorem 2.4]) is that if for any maximal ideal $\mathfrak{m} \subset R$, the group $\mathcal{G}_{R_{\mathfrak{m}}}$ is isotropic of rank $\geq 2$, then $E_{P}(R)$ does not depend on the choice of a strictly parabolic subgroup $P$. This assumption is automatically satisfied in all situations considered in this paper, so, to simplify notations, we will denote the elementary subgroup simply by $E(R)$.

Furthermore, in analogy with elementary subgroups of Chevalley groups, Petrov and Stavrova provide a description of $E(R)$ in terms of generators that satisfy certain generalized Chevalley commutator relations. The following statement collects the relevant parts of [13, Theorem 2] and [22, Lemma 2.14] in the case of $G=\mathrm{SU}_{2 n}(L, h)$ that will be needed for our analysis.

Theorem 2.1. Let $G=\mathrm{SU}_{2 n}(L, h)$, fix a maximal $k$-split torus $S \subset G$, and denote by $\Phi_{k}$ the corresponding relative root system (of type $\mathrm{C}_{n}$ ), viewed as a subset of the character group $X^{*}(S)$. Then for every $\alpha \in \Phi_{k}$, there exists a vector $k$-group scheme $V_{\alpha}$ and a closed embedding of schemes

$$
X_{\alpha}: V_{\alpha} \rightarrow G
$$

such that for any $k$-algebra $R$, we have the following:

(1) For any $v, w \in V_{\alpha}(R)$,

$$
X_{\alpha}(v) \cdot X_{\alpha}(w)=X_{\alpha}(v+w)
$$

In particular, $X_{\alpha}(0)=1$.

(2) For any $s \in S(R)$ and $v \in V_{\alpha}(R)$,

$$
s \cdot X_{\alpha}(v) \cdot s^{-1}=X_{\alpha}(\alpha(s) v)
$$

(3) (Chevalley commutator formula) For any $\alpha, \beta \in \Phi_{k}$ such that $\alpha \neq \pm \beta$, and for all $u \in$ $V_{\alpha}(R), v \in V_{\beta}(R)$

$$
\left[X_{\alpha}(u), X_{\beta}(v)\right]=\prod_{\substack{i, j \geq 1 \\ i \alpha+j \beta \in \Phi_{k}}} X_{i \alpha+j \beta}\left(N_{i j}^{\alpha \beta}(u, v)\right)
$$

for some polynomial maps $N_{i j}^{\alpha \beta}: V_{\alpha}(R) \times V_{\beta}(R) \rightarrow V_{i \alpha+j \beta}(R)$. The map $N_{i j}^{\alpha \beta}$ is homogeneous of degree $i$ in the first variable and homogeneous of degree $j$ in the second variable. 
(4) The elementary subgroup $E(R)$ is generated by the elements $X_{\alpha}(v)$ for all $\alpha \in \Phi_{k}$ and all $v \in V_{\alpha}(R)$.

\section{Remark 2.2.}

(a) In view of the structure of the root system $C_{n}$, it is easy to see that the products on the right hand side of (3) contain at most two terms with the possible values of $i$ and $j$ lying in $\{1,2\}$. Moreover, in the cases where there are two terms, these commute with each other.

(b) It follows from the definitions that the construction of the elementary subgroup is functorial in $R$ (i.e. a ring homomorphism $R_{1} \rightarrow R_{2}$ gives rise to a group homomorphism $E\left(R_{1}\right) \rightarrow E\left(R_{2}\right)$ ), and that it is compatible with finite products (i.e. $\left.E\left(R_{1} \times \cdots \times R_{n}\right)=E\left(R_{1}\right) \times \cdots \times E\left(R_{n}\right)\right)$. Furthermore, since $G=\mathrm{SU}_{2 n}(L, h)$ is a quasi-split simply-connected $k$-group, this observation and [22, Lemma 5.2] imply that $G(R)=E(R)$ for any ring $R$ that is a finite product of local $k$-algebras.

Continuing with the notations of the theorem, the dimension of $V_{\alpha}$ is simply the dimension of the relative root space associated to $\alpha$. Thus, in our situation, there are two possibilities: if $\alpha$ is a long root, then $V_{\alpha}=\mathbb{G}_{a}$, so that $V_{\alpha}(R)=R$ for any $k$-algebra $R$; on the other hand, if $\alpha$ is short, then $V_{\alpha} \cong\left(\mathbb{G}_{a}\right)^{2}$, and we have $V_{\alpha}(R)=R \otimes_{k} L=R_{L}$ (to make the identification $\left(\mathbb{G}_{a}\right)^{2}(R)=R^{2} \cong R_{L}$, we use the fact that $k^{2} \cong L$ as a $k$-vector space).

For the calculations that we will carry out in subsequent sections, it will be useful to make the statement of Theorem 2.1 more explicit, as follows. Fix an algebraic closure $\bar{k}$ of $k$ and consider the maximal $k$-split torus $S$ of $G$ (we will identify $G$ with $G(\bar{k})$ for the sake of concreteness) consisting of diagonal matrices of the form

$$
\left(\begin{array}{ccccc}
t_{1} & & & & \\
& t_{1}^{-1} & & & \\
& & \ddots & & \\
& & & t_{n} & \\
& & & & t_{n}^{-1}
\end{array}\right)
$$

with $t_{i} \in \bar{k}$. For $1 \leq i \leq 2 n$, let $\alpha_{i}: S \rightarrow \mathbb{G}_{m}$ be the character that maps such a matrix to its $i$-th diagonal entry. Then one easily checks that

$$
\Phi_{k}=\left\{ \pm 2 \alpha_{i}: i \text { odd }\right\} \cup\left\{ \pm \alpha_{i} \pm \alpha_{j}: i \neq j \text {, both odd }\right\}
$$

with $1 \leq i, j \leq 2 n$. Furthermore, the morphisms $X_{\alpha}$ look as follows. For a ring $R$, we denote by $E_{i j}(x) \in \mathrm{M}_{2 n}(R)$ the matrix with $x$ in the $i j$-th entry and 0 in all other entries. Also, if $R$ is a $k$-algebra, then, as we observed previously, the action of the nontrivial element $\tau \in \operatorname{Gal}(L / k)$ extends to $R_{L}=R \otimes_{k} L$, and we will write $\tau(a)=\bar{a}$ for $a \in R_{L}$. Then the root group morphisms for the long roots are

$$
\begin{aligned}
X_{2 \alpha_{i}}(R): R \rightarrow G(R) & x \mapsto 1+E_{i, i+1}(x) \\
X_{-2 \alpha_{i}}(R): R \rightarrow G(R) & x \mapsto X_{2 \alpha_{i}}(x)^{t}=1+E_{i+1, i}(x),
\end{aligned}
$$

and for the short roots, the morphisms are

$$
\begin{aligned}
X_{\alpha_{i}-\alpha_{j}}(R): R_{L} \rightarrow G(R) & x \mapsto 1+E_{i j}(x)-E_{j+1, i+1}(\bar{x}) \\
X_{-\alpha_{i}+\alpha_{j}}(R): R_{L} \rightarrow G(R) & x \mapsto X_{\alpha_{i}-\alpha_{j}}(x)^{t}=1+E_{j i}(x)-E_{i+1, j+1}(\bar{x}), \\
X_{\alpha_{i}+\alpha_{j}}(R): R_{L} \rightarrow G(R) & x \mapsto 1+E_{i^{\prime}, j^{\prime}+1}(x)+E_{j^{\prime}, i^{\prime}+1}(\bar{x}), \\
X_{-\alpha_{i}-\alpha_{j}}(R): R_{L} \rightarrow G(R) & x \mapsto X_{\alpha_{i}+\alpha_{j}}(x)^{t}=1+E_{j^{\prime}+1, i^{\prime}}(x)+E_{i^{\prime}+1, j^{\prime}}(\bar{x}),
\end{aligned}
$$

where for a pair $i, j$, we set $i^{\prime}=\min (i, j)$ and $j^{\prime}=\max (i, j)$.

By direct calculation, one obtains explicit formulas for the polynomial maps $N_{i j}^{\alpha \beta}$ appearing in Theorem 2.1. To formulate the result, given a $k$-algebra $R$, we let

$$
\operatorname{Tr}: R_{L} \rightarrow R \quad a \mapsto a+\bar{a}
$$


denote the extension of the usual trace map $\operatorname{Tr}_{L / k}: L \rightarrow k$ to $R_{L}$. Also, for $\delta= \pm 1$ and $v \in R_{L}$, we set

$$
v_{\delta}= \begin{cases}v & \delta=1 \\ \bar{v} & \delta=-1\end{cases}
$$

The following lemma describes the maps $N_{i j}^{\alpha \beta}$ arising in all nontrivial Chevalley commutator relations for $\mathrm{SU}_{2 n}(L, h)$.

Lemma 2.3. Let $\alpha, \beta \in \Phi_{k}$ be relative roots such that $\alpha+\beta \in \Phi_{k}$ and let $R$ be a $k$-algebra.

(1) Suppose $\alpha$ and $\beta$ are short and $\alpha+\beta$ is short. Then (relabelling if necessary) we can write $\alpha=\alpha_{i}-\alpha_{j}$ and $\beta=\alpha_{j}-\alpha_{\ell}$ for three distinct indices $i, j, \ell$, and

$$
N_{11}^{\alpha \beta}(u, v)=u v \quad N_{11}^{\beta \alpha}(v, u)=-u v
$$

(2) Suppose $\alpha$ and $\beta$ are both short and $\alpha+\beta$ is long. Then (relabelling if necessary) we can write $\alpha=\epsilon\left(\alpha_{i}-\alpha_{j}\right), \beta=\omega\left(\alpha_{i}+\alpha_{j}\right)$ for some $\epsilon= \pm 1, \omega= \pm 1$, with $i<j$, and

$$
N_{11}^{\alpha \beta}(u, v)=\omega \operatorname{Tr}\left(u_{-\epsilon \omega} v\right) \quad N_{11}^{\beta \alpha}(v, u)=-\omega \operatorname{Tr}\left(u_{-\epsilon \omega} v\right)
$$

for all $u \in V_{\alpha}(R), v \in V_{\beta}(R)$.

(3) Suppose $\alpha$ is short and $\beta$ long. Then we can write $\alpha=\epsilon \alpha_{i}+\omega \alpha_{j}$ and $\beta=-\epsilon 2 \alpha_{i}$ for some $\epsilon= \pm 1, \omega= \pm 1$ and $i \neq j$, and

$$
\begin{array}{ll}
N_{11}^{\alpha \beta}(u, v)=\omega u_{-c_{i j}} v & N_{11}^{\beta \alpha}(v, u)=-\omega u_{-c_{i j}} v \\
N_{21}^{\alpha \beta}(u, v)=-\epsilon \omega v u \bar{u} & N_{12}^{\beta \alpha}(v, u)=\epsilon \omega v u \bar{u}
\end{array}
$$

for all $u \in V_{\alpha}(R), v \in V_{\beta}(R)$, where

$$
c_{i j}= \begin{cases}1 & i<j \\ -1 & i>j\end{cases}
$$

In particular, whenever it is defined, the map $N_{11}^{\alpha \beta}$ is surjective.

Next, as we indicated in \$1, a key step in the proof of our main result involves restricting a given representation $\rho: G(k) \rightarrow \mathrm{GL}_{m}(K)$ to the subgroup $G_{0}(k)=\operatorname{Sp}_{2 n}(k)$. Our choice of $H$ makes this split subgroup simple to describe. It is

$$
G(k) \cap \mathrm{GL}_{2 n}(k)=\left\{X \in \mathrm{SL}_{2 n}(k) \mid X^{t} H X=H\right\}=\operatorname{Sp}_{2 n}(k)=G_{0}(k) .
$$

Now, recall that if $\beta \in \Phi_{k}$ is a short root, then $V_{\beta}(k)=L=k \oplus k \sqrt{d}$. Our calculation then shows that $G_{0}(k)$ is the subgroup of $G(k)=E(k)$ generated by the images of the maps $X_{\alpha}(k): V_{\alpha}(k) \rightarrow G(k)$ for long roots $\alpha$ and by the images of the maps $X_{\beta}(k): V_{\beta}(k) \rightarrow G(k)$ restricted to the first component for long roots $\beta$.

For later use, following Steinberg (see [24, Ch. 3]), we define the elements

$$
w_{\alpha}(v)=X_{\alpha}(v) \cdot X_{-\alpha}\left(-v^{-1}\right) \cdot X_{\alpha}(v) \quad \text { and } \quad h_{\alpha}(v)=w_{\alpha}(v) \cdot w_{\alpha}(1)^{-1}
$$

for $\alpha \in \Phi_{k}$ and $v \in V_{\alpha}(k)^{\times}$(thus, in particular, $w_{\alpha}(1), h_{\alpha}(1) \in G_{0}(k)$ ). Then we have the following analogue of Steinberg's relation (R7)

$$
w_{\alpha}(1) \cdot X_{\beta}(v) \cdot w_{\alpha}(1)^{-1}=X_{w_{\alpha} \beta}(\varphi v),
$$

where $\varphi v= \pm v_{ \pm 1}$ (so that $\left.\varphi^{2}=\mathrm{Id}\right)$ and $w_{\alpha} \beta$ denotes the action of the corresponding Weyl group element $w_{\alpha}$ on the root $\beta$. 
2.2. Steinberg groups. In this subsection, we recall Stavrova's [22] generalization of the usual Steinberg group (which, in turn, is inspired by a construction of Deodhar [8]) and establish several properties that will be needed in subsequent sections. Although Stavrova works in the general context of reductive group schemes, for the sake of concreteness, we will restrict ourselves to the case $G=\mathrm{SU}_{2 n}(L, h)$.

As in the classical setting, for a $k$-algebra $R$, the generalized Steinberg group $\widetilde{G}(R)$ is the abstract group generated by symbols $\widetilde{X}_{\alpha}(v)$, for $\alpha \in \Phi_{k}$ and $v \in V_{\alpha}(R)$, subject to the relations in Theorem 2.1 written in terms of the $\widetilde{X}_{\alpha}$. More precisely, we have the following.

Definition 2.4. Let $R$ be a $k$-algebra. We define $\widetilde{G}(R)$ to be the group generated by symbols $\widetilde{X}_{\alpha}(v)$, for all $\alpha \in \Phi_{k}$ and all $v \in V_{\alpha}(R)$, subject to the following relations:

(R1) For $\alpha \in \Phi_{k}$ and $v, w \in V_{\alpha}(R)$,

$$
\widetilde{X}_{\alpha}(v) \cdot \widetilde{X}_{\alpha}(w)=\widetilde{X}_{\alpha}(v+w)
$$

(R2) For $\alpha, \beta \in \Phi_{k}$ such that $\alpha \neq \pm \beta$, and for all $u \in V_{\alpha}(R), v \in V_{\beta}(R)$,

$$
\left[\widetilde{X}_{\alpha}(u), \widetilde{X}_{\beta}(v)\right]=\prod_{\substack{i, j \geq 1 \\ i \alpha+j \beta \in \Phi_{k}}} \widetilde{X}_{i \alpha+j \beta}\left(N_{i j}^{\alpha \beta}(u, v)\right) ;
$$

where $N_{i j}^{\alpha \beta}$ are the same maps as in Theorem $2.1(3)$.

It is clear that this construction is functorial in $R$. Furthermore, for every $k$-algebra $R$, we have a natural surjective homomorphism

$$
\pi_{R}: \widetilde{G}(R) \rightarrow E(R) \quad \widetilde{X}_{\alpha}(v) \mapsto X_{\alpha}(v) .
$$

The main result of this subsection is the following statement, which partially extends [22, Theorem $1.3]$.

Proposition 2.5. Suppose $R$ is a k-algebra that is a finite product of local k-algebras. Then ker $\pi_{R}$ is a central subgroup of $\widetilde{G}(R)$.

We will give the proof of Proposition 2.5 below, after several preliminary observations.

Lemma 2.6. Any generator $\widetilde{X}_{\alpha}(v)$ of $\widetilde{G}(R)$ can be written as a product of generators $\widetilde{X}_{\gamma_{i}}\left(u_{i}\right)$ with $\gamma_{i} \neq \alpha$. Furthermore, $\widetilde{X}_{\alpha}(v)$ is contained in the commutator subgroup $[\widetilde{G}(R), \widetilde{G}(R)]$. In particular, $\widetilde{G}(R)$ and $E(R)$ are perfect groups.

Proof. First, suppose $\alpha$ is a long root. Then we can write $\alpha=\gamma_{1}+\gamma_{2}$ for appropriate short roots $\gamma_{1}$ and $\gamma_{2}$, and it follows from (R2) that

$$
\left[\widetilde{X}_{\gamma_{1}}\left(u_{1}\right), \widetilde{X}_{\gamma_{2}}\left(u_{2}\right)\right]=\widetilde{X}_{\alpha}\left(N_{11}^{\gamma_{1} \gamma_{2}}\left(u_{1}, u_{2}\right)\right)
$$

Since $N_{11}^{\gamma_{1} \gamma_{2}}$ is surjective according to Lemma 2.3, we can find $u_{1} \in V_{\gamma_{1}}(R)$ and $u_{2} \in V_{\gamma_{2}}(R)$ so that $N_{11}^{\gamma_{1} \gamma_{2}}\left(u_{1}, u_{2}\right)=v$, which yields our claim in this case.

Next, suppose $\alpha$ is short. Then we can write $\alpha=\gamma_{1}+\gamma_{2}$ for an appropriate long root $\gamma_{1}$ and short root $\gamma_{2}$. Hence, by (R2) we have

$$
\left[\widetilde{X}_{\gamma_{1}}\left(u_{1}\right), \widetilde{X}_{\gamma_{2}}\left(u_{2}\right)\right]=\widetilde{X}_{\alpha}\left(N_{11}^{\gamma_{1} \gamma_{2}}\left(u_{1}, u_{2}\right)\right) \cdot \widetilde{X}_{\gamma_{1}+2 \gamma_{2}}\left(N_{12}^{\gamma_{1} \gamma_{2}}\left(u_{1}, u_{2}\right)\right)
$$

Again by Lemma 2.3 we can choose $u_{1}, u_{2}$ so that $N_{11}^{\gamma_{1} \gamma_{2}}\left(u_{1}, u_{2}\right)=v$. Multiplying both sides by $\widetilde{X}_{\gamma_{1}+2 \gamma_{2}}\left(N_{12}^{\gamma_{1} \gamma_{2}}\left(u_{1}, u_{2}\right)\right)^{-1}$ then yields our first claim. Furthermore, since $\gamma_{1}+2 \gamma_{2}$ is a long root, the preceding case shows that $\widetilde{X}_{\gamma_{1}+2 \gamma_{2}}\left(N_{12}^{\gamma_{1} \gamma_{2}}\left(u_{1}, u_{2}\right)\right)^{-1}$ is contained in the commutator subgroup, and hence so is $\widetilde{X}_{\alpha}(v)$.

Thus, since all generators of $\widetilde{G}(R)$ are contained in $[\widetilde{G}(R), \widetilde{G}(R)]$, it follows that $\widetilde{G}(R)$ is perfect. Since the natural map $\pi: \widetilde{G}(R) \rightarrow E(R)$ is surjective, we conclude that $E(R)$ is perfect as well. 
Lemma 2.7. For any $k$-algebras $R_{1}, \ldots, R_{n}$, we have $\widetilde{G}\left(R_{1} \times \cdots \times R_{n}\right)=\widetilde{G}\left(R_{1}\right) \times \cdots \times \widetilde{G}\left(R_{n}\right)$, i.e. the Steinberg group commutes with finite products.

Proof. By induction, it suffices to show that $\widetilde{G}(A \times B) \cong \widetilde{G}(A) \times \widetilde{G}(B)$ for any $k$-algebras $A$ and $B$. We will do this by an argument similar to the one used in the proof of [23, Lemma 2.12]. First, we note that the projections of $A \times B$ onto its components induce a surjective group homomorphism

$$
p: \widetilde{G}(A \times B) \rightarrow \widetilde{G}(A) \times \widetilde{G}(B), \quad \widetilde{X}_{\alpha}(v)=\widetilde{X}_{\alpha}\left(v_{1}, v_{2}\right) \mapsto\left(\widetilde{X}_{\alpha}\left(v_{1}\right), \widetilde{X}_{\alpha}\left(v_{2}\right)\right)
$$

Next we define a map in the reverse direction by

$$
\begin{aligned}
s: \widetilde{G}(A) \times \widetilde{G}(B) & \rightarrow \widetilde{G}(A \times B) \\
\left(\widetilde{X}_{\alpha}\left(v_{1}\right), 1\right) & \mapsto \widetilde{X}_{\alpha}\left(v_{1}, 0\right) \\
\left(1, \widetilde{X}_{\alpha}\left(v_{2}\right)\right) & \mapsto \widetilde{X}_{\alpha}\left(0, v_{2}\right)
\end{aligned}
$$

(This defines $s$ on a set of generators for $\widetilde{G}(A) \times \widetilde{G}(B)$, and we then extend it to the whole group by multiplicativity.) It follows immediately from the definitions that $p \circ s$ and $s \circ p$ are the respective identity maps on generating sets. Thus, it remains to show that $s$ is a homomorphism by verifying that $s$ takes all the defining relations in $\widetilde{G}(A) \times \widetilde{G}(B)$ to relations in $\widetilde{G}(A \times B)$. We need to check that $s$ preserves three kinds of relations:

(i) The defining relations of $\widetilde{G}(A)$ applied to the generators $\left(\widetilde{X}_{\alpha}\left(v_{1}\right), 1\right)$.

(ii) The defining relations of $\widetilde{G}(B)$ applied to the generators $\left(1, \widetilde{X}_{\alpha}\left(v_{2}\right)\right)$.

(iii) $\left[\left(\widetilde{X}_{\alpha}(a), 1\right),\left(1, \widetilde{X}_{\beta}(b)\right)\right]=1$ for all $\alpha, \beta \in \Phi_{k}$ and all $a \in V_{\alpha}(A), b \in V_{\beta}(B)$.

It is clear that $s$ preserves (i) and (ii), so it remains to show that $s$ preserves (iii). First consider the case $\alpha \neq-\beta$. Let $a \in V_{\alpha}(A)$ and $b \in V_{\beta}$. Then

$$
s\left[\left(\widetilde{X}_{\alpha}(a), 1\right),\left(1, \widetilde{X}_{\beta}(b)\right)\right]=\left[\widetilde{X}_{\alpha}(a, 0), \widetilde{X}_{\beta}(0, b)\right]=\prod_{\substack{i, j \geq 1 \\ i \alpha+j \beta \in \Phi_{k}}} X_{i \alpha+j \beta}\left(N_{i j}^{\alpha \beta}((a, 0),(0, b))\right)
$$

Since $N_{i j}^{\alpha \beta}$ is homogeneous of degree $i$ in the first argument and of degree $j$ in the second argument, it is at least linear in each argument. So each term of $N_{i j}^{\alpha \beta}((a, 0),(0, b))$ includes a factor of $(a, 0) \cdot(0, b)=$ $(0,0)=0 \in A \times B$. Hence $N_{i j}^{\alpha \beta}((a, 0),(0, b))=0$ and the product is trivial, so $s$ preserves (iii) when $\alpha \neq-\beta$.

Now consider relation (iii) with $\alpha=-\beta$. For any $b \in B$, by Lemma 2.6, the element $\widetilde{X}_{-\alpha}(0, b) \in$ $\widetilde{G}(0 \times B) \subset \widetilde{G}(A \times B)$ can be written as a product

$$
\widetilde{X}_{-\alpha}(0, b)=\prod_{i} \tilde{X}_{\gamma_{i}}\left(0, u_{i}\right)
$$

where $\gamma_{i} \neq-\alpha$ for all $i$, with each $u_{i} \in B$. Thus, we have

$$
s\left[\left(\widetilde{X}_{\alpha}(a), 1\right),\left(1, \widetilde{X}_{-\alpha}(b)\right)\right]=\left[\widetilde{X}_{\alpha}(a, 0), \widetilde{X}_{-\alpha}(0, b)\right]=\left[\widetilde{X}_{\alpha}(a, 0), \prod_{i} \widetilde{X}_{\gamma_{i}}\left(0, u_{i}\right)\right]
$$

Since $\gamma_{i} \neq-\alpha$, by the previous case, $\widetilde{X}_{\alpha}(a, 0)$ commutes with each factor $\widetilde{X}_{\gamma_{i}}\left(0, u_{i}\right)$. Consequently, it commutes with the whole product, hence the last commutator vanishes. This shows that $s$ preserves (iii) when $\alpha=-\beta$, completing the proof.

We now turn to:

Proof of Proposition 2.5. Suppose $R=R_{1} \times \cdots \times R_{n}$, where $R_{1}, \ldots, R_{n}$ are local $k$-algebras. Since the elementary subgroup and the Steinberg group both commute with finite products (by Remark 2.2 
and Lemma 2.7, respectively), it follows that

$$
\pi_{R}=\prod_{i=1}^{n} \pi_{R_{i}}
$$

and hence

$$
\operatorname{ker} \pi_{R}=\prod_{i=1}^{n} \operatorname{ker} \pi_{R_{i}} \subset \prod_{i=1}^{n} \widetilde{G}\left(R_{i}\right) \cong \widetilde{G}(R) .
$$

Applying [22, Theorem 1.3] to each local factor $R_{i}$, we see that $\operatorname{ker} \pi_{R_{i}}$ is central in $\widetilde{G}\left(R_{i}\right)$. Consequently, $\operatorname{ker} \pi_{R}$ is central in $\widetilde{G}(R)$, as claimed.

\section{Constructing the algebraic ring $A$ and lifting $\rho$ to a Representation of the STEINBERG GROUP}

Let $G=\mathrm{SU}_{2 n}(L, h)$ and set $G_{0}(k)=\mathrm{Sp}_{2 n}(k)$ to be the split subgroup of $G(k)$ described in (11). The purpose of this section is twofold. First, we associate an algebraic ring $A$ to an abstract representation $\rho: G(k) \rightarrow \mathrm{GL}_{m}(K)$. Then we show that $\rho$ can be lifted to a representation $\widetilde{\sigma}$ of the Steinberg group $\widetilde{G}(A)$.

To fix notations, given an abstract representation $\rho: G(k) \rightarrow \mathrm{GL}_{m}(K)$ (with $K$ an algebraically closed field of characteristic 0 ), we set $H=\overline{\rho(G(k))}$ to be the Zariski closure of the image of $\rho$. Furthermore, we let $\rho_{0}: G_{0}(k) \rightarrow \mathrm{GL}_{m}(K)$ denote the restriction $\left.\rho\right|_{G_{0}(k)}$.

By [16, Theorem 3.1], we can associate to $\rho_{0}$ an algebraic ring $A$, together with a ring homomorphism $f: k \rightarrow A$ with Zariski-dense image, as follows. Recall that if $\alpha \in \Phi_{k}$ is a long root, then $V_{\alpha}(k)=k$, whereas if $\alpha$ is short, then $V_{\alpha}(k)=L=k \oplus k \sqrt{d}$. For $\alpha \in \Phi_{k}$, we define

$$
A_{\alpha}=\overline{\rho\left(X_{\alpha}(k)\right)}
$$

and

$$
f_{\alpha}: k \rightarrow A_{\alpha}, \quad u \mapsto \rho_{0}\left(X_{\alpha}(u)\right) .
$$

Recall that for $\alpha$ short, the root subgroup $X_{\alpha}\left(V_{\alpha}(k)\right)$ has dimension 2, and in this case, $A_{\alpha}$ is the closure of the image of the one-dimensional subgroup $X_{\alpha}(k) \subset X_{\alpha}\left(V_{\alpha}(k)\right)$, arising from the natural embedding $k \hookrightarrow L=V_{\alpha}(k)$.

As shown in [16, Theorem 3.1], each $A_{\alpha}$ has the structure of an algebraic ring (i.e. an affine algebraic variety with a ring structure defined by regular maps) — we recall that the addition operation is obtained simply by restricting matrix multiplication in $H$ to $A_{\alpha}$, whereas the multiplication operation is defined using the Steinberg commutator relations. Moreover, for any $\alpha, \beta \in \Phi_{k}$, there exists an isomorphism $\pi_{\alpha \beta}: A_{\alpha} \rightarrow A_{\beta}$ of algebraic rings such that $\pi_{\alpha \beta} \circ f_{\alpha}=f_{\beta}$. We denote this common algebraic ring by $A$, and, for each $\alpha \in \Phi_{k}$, we fix an isomorphism of algebraic rings $\pi_{\alpha}: A \rightarrow A_{\alpha}$ such that $\pi_{\alpha \beta} \circ \pi_{\alpha}=\pi_{\beta}$. Then, by construction, we have a ring homomorphism $f: k \rightarrow A$ with Zariskidense image such that $\pi_{\alpha} \circ f=f_{\alpha}$ for all $\alpha \in \Phi_{k}$, as well as (injective) regular maps $\psi_{\alpha}^{1}: A \rightarrow H$ satisfying

$$
\left(\psi_{\alpha}^{1} \circ f\right)(u)=\left(\rho_{0} \circ X_{\alpha}\right)(u) \text { for all } u \in k .
$$

Remark 3.1. Since $k$ and $K$ are both fields of characteristic 0, it follows from Lemma 2.13 and Proposition 2.14 in [16] that $A$ is a finite-dimensional $K$-algebra. In particular, $A$ is connected and satisfies the hypotheses of Proposition 2.5.

The algebraic ring $A$ plays a pivotal role in the proof of the main results of [16]. It turns out that $A$ also suffices for the analysis of the representation $\rho$. The precise statement that is needed in our context will be given in Proposition 3.5 below. First, we make the following construction. Given a short root $\alpha \in \Phi_{k}$, let us define

and

$$
B_{\alpha}=\overline{\rho\left(X_{\alpha}(k \sqrt{d})\right)}
$$

$$
g_{\alpha}: k \rightarrow B_{\alpha}, \quad u \mapsto \rho\left(X_{\alpha}(u \sqrt{d})\right)
$$


We then have the next statement.

Lemma 3.2. Take the short root $\alpha=\alpha_{1}-\alpha_{3}$ and the long root $\beta=-2 \alpha_{3}$. Then there is an isomorphism of algebraic varieties $\pi: B_{\alpha} \rightarrow A_{\beta}$ such that $\pi \circ g_{\alpha}=f_{\beta}$.

Proof. (cf. [16, Lemma 3.3]) By Lemma 2.3(1), we have $N_{11}^{\alpha, \beta-\alpha}(u, v)=-\operatorname{Tr}(u v)$. Define a regular $\operatorname{map} \pi: B_{\alpha} \rightarrow H$ by

$$
\pi(x)=\left[x, g_{\beta-\alpha}\left(\frac{-1}{2 d}\right)\right]
$$

Now let $s \in k$. Then

$$
\begin{aligned}
\pi \circ g_{\alpha}(s) & =\left[\rho \circ X_{\alpha}(s \sqrt{d}), \rho \circ X_{\beta-\alpha}\left(\frac{-\sqrt{d}}{2 d}\right)\right]=\rho\left[X_{\alpha}(s \sqrt{d}), X_{\beta-\alpha}\left(\frac{-1}{2 \sqrt{d}}\right)\right] \\
& =\rho \circ X_{\beta}\left(N_{11}^{\alpha, \beta-\alpha}\left(s \sqrt{d}, \frac{-1}{2 \sqrt{d}}\right)\right)=\rho \circ X_{\beta}\left(-\operatorname{Tr}\left(\frac{-s}{2}\right)\right)=\rho\left(X_{\beta}(s)\right)=f_{\beta}(s)
\end{aligned}
$$

This shows that $\pi \circ g_{\alpha}=f_{\beta}$. Since $\pi$ is regular, it then follows that $\pi\left(B_{\alpha}\right) \subset A_{\beta}$. It remains to show that $\pi$ is invertible. First, using Lemma 2.3(2), we obtain

$$
\begin{aligned}
& N_{11}^{\beta, \alpha-\beta}(v, u)=-u v \\
& N_{12}^{\beta, \alpha-\beta}(v, u)=v u \bar{u}
\end{aligned}
$$

Now let $h=h_{2 \alpha-\beta}(1 / 2)$ be the element introduced in (2) and define

$$
\nu: A_{\beta} \rightarrow B_{\alpha}, \quad \nu(y)=\rho(h) \cdot\left[y, g_{\alpha-\beta}(-1)\right] \cdot\left[y, g_{\alpha-\beta}(1)\right]^{-1} \cdot \rho(h)^{-1} .
$$

It is clear that $\nu$ is a regular map; we claim it is an inverse for $\pi$. Let $t \in k$. Using the commutator relation in Theorem 2.1(3), we have

$$
\begin{aligned}
{\left[X_{\beta}(t), X_{\alpha-\beta}(-\sqrt{d})\right] } & =X_{\alpha}\left(N_{11}^{\beta, \alpha-\beta}(t,-\sqrt{d})\right) \cdot X_{2 \alpha-\beta}\left(N_{12}^{\beta, \alpha-\beta}(t,-\sqrt{d})\right) \\
& =X_{\alpha}(t \sqrt{d}) \cdot X_{2 \alpha-\beta}(-t d) .
\end{aligned}
$$

Thus

$$
\begin{aligned}
& {\left[X_{\beta}(t), X_{\alpha-\beta}(-\sqrt{d})\right] \cdot\left[X_{\beta}(t), X_{\alpha-\beta}(\sqrt{d})\right]^{-1}} \\
& =X_{\alpha}(t \sqrt{d}) \cdot X_{2 \alpha-\beta}(-t d) \cdot X_{2 \alpha-\beta}(-t d)^{-1} \cdot X_{\alpha}(-t \sqrt{d})^{-1}=X_{\alpha}(2 t \sqrt{d}) .
\end{aligned}
$$

We also have the relation

$$
h \cdot X_{\alpha}(2 v) \cdot h^{-1}=X_{\alpha}(v)
$$

for all $v \in L$. Putting everything together, we obtain

$$
\begin{aligned}
\nu \circ f_{\beta}(t) & =\rho\left(h \cdot\left[X_{\beta}(t), X_{\alpha-\beta}(-\sqrt{d})\right] \cdot\left[X_{\beta}(t), X_{\alpha-\beta}(\sqrt{d})\right]^{-1} \cdot h^{-1}\right) \\
& =\rho\left(h \cdot X_{\alpha}(2 t \sqrt{d}) \cdot h^{-1}\right)=\rho\left(X_{\alpha}(t \sqrt{d})\right)=g_{\alpha}(t) .
\end{aligned}
$$

Thus, $\nu \circ \pi$ and $\pi \circ \nu$ are the respective identity maps on dense subsets of $A_{\beta}$ and $B_{\alpha}$. Since they are regular, it follows that they are the respective identities on the whole space, so $\nu$ is the inverse of $\pi$ as claimed.

Remark 3.3. Although we fixed the roots $\alpha$ and $\beta$ in the preceding argument for the sake of concreteness, essentially the same calculations can be carried for any short root $\alpha$ and long root $\beta$ such that $\alpha-\beta$ is a root (with appropriate modifications to the definitions of $\pi$ and $\nu$, depending on the signs arising in computing $N_{11}$ and $N_{12}$ ). 
Next, we make the following observation, which will streamline the proof of Proposition 3.5 by allowing us to consider just a single root of each length. In the statement, we refer to the group schemes $V_{\alpha}$ introduced in Theorem 2.1. Given a $k$-algebra homomorphism $f: k \rightarrow A$, we denote by $V_{\alpha}(f): V_{\alpha}(k) \rightarrow V_{\alpha}(A)$ the associated group homomorphism (note that if $\alpha$ is a long root, then $V_{\alpha}(f)$ can be identified with $f$, and if $\alpha$ is short, then $V_{\alpha}(f)$ is the map $a+b \sqrt{d} \mapsto f(a)+f(b) \sqrt{d}-$ in particular, if $\alpha$ and $\beta$ have the same length, then the homomorphisms $V_{\alpha}(f)$ and $V_{\beta}(f)$ coincide).

Lemma 3.4. Let $\alpha, \beta \in \Phi_{k}$ be roots of the same length. Then

(1) There exists $w \in E(k)$ such that for all $v \in V_{\alpha}(k)$, we have $w \cdot X_{\alpha}(v) \cdot w^{-1}=X_{\beta}(\varphi v)$, where $\varphi v= \pm v_{ \pm 1}$.

(2) Let $f: k \rightarrow A$ be a $k$-algebra homomorphism. Suppose there exists a regular map $\psi_{\alpha}: V_{\alpha}(A) \rightarrow$ $H$ such that $\psi_{\alpha} \circ V_{\alpha}(f)=\rho \circ X_{\alpha}$. Let $w, \varphi$ be as in (1), and define $\psi_{\beta}: V_{\beta}(A) \rightarrow H$ by

$$
\psi_{\beta}(v)=\rho(w) \cdot \psi_{\alpha}(\varphi v) \cdot \rho(w)^{-1}
$$

Then $\psi_{\beta}$ is regular and satisfes $\psi_{\beta} \circ V_{\beta}(f)=\rho \circ X_{\beta}$.

Proof. (1) It is well-known that the Weyl group $W$ acts transitively on roots of the same length, so there exists $\widetilde{w} \in W$ such that $\widetilde{w} \alpha=\beta$. Write $\widetilde{w}$ as a product of simple reflections, $\widetilde{w}=w_{\gamma_{1}} \cdots w_{\gamma_{n}}$ for roots $\gamma_{i} \in \Phi_{k}$. Using the relation (3), we have

$$
w_{\gamma_{i}}(1) \cdot X_{\alpha}(v) \cdot w_{\gamma_{i}}(1)^{-1}=X_{w_{\gamma_{i}} \alpha}\left(\varphi_{i} v\right)
$$

where $\varphi_{i} v= \pm v_{ \pm 1}$. Let $w=w_{\gamma_{1}}(1) \cdots w_{\gamma_{n}}(1)$ and $\varphi=\varphi_{1} \cdots \varphi_{n}$. Then repeatedly applying the above relation yields

$$
w \cdot X_{\alpha}(v) \cdot w^{-1}=X_{w_{\gamma_{1}} \cdots w_{\gamma_{n}} \alpha}\left(\varphi_{1} \cdots \varphi_{n} v\right)=X_{\widetilde{w} \alpha}(\varphi v)=X_{\beta}(\varphi v)
$$

Since each $\varphi_{i}$ is a composition of negation and conjugation, $\varphi v= \pm v_{ \pm 1}$.

(2) This is proved by a direct calculation using the result of part (1), the fact that $\varphi^{2}=\mathrm{Id}$, and the assumption that $\psi_{\alpha} \circ V_{\alpha}(f)=\rho \circ X_{\alpha}$. Namely, let $v \in V_{\beta}(A)$. Then

$$
\begin{aligned}
\psi_{\beta} \circ V_{\beta}(f)(v) & =\rho(w) \cdot \psi_{\alpha}\left(\varphi \circ V_{\beta}(f)(v)\right) \cdot \rho(w)^{-1}=\rho(w) \cdot \psi_{\alpha} \circ V_{\alpha}(f)(\varphi v) \cdot \rho(w)^{-1}= \\
& =\rho(w) \cdot \rho \circ X_{\alpha}(\varphi v) \cdot \rho(w)^{-1}=\rho\left(w \cdot X_{\alpha}(\varphi v) \cdot w^{-1}\right)=\rho\left(X_{\beta}\left(\varphi^{2} v\right)\right)=\rho \circ X_{\beta}(v)
\end{aligned}
$$

We now come to one of the main statements of this section.

Proposition 3.5. Let $G=\mathrm{SU}_{2 n}(L, h)$ and let $\rho: G(k) \rightarrow \mathrm{GL}_{m}(K)$ be an abstract representation, with $K$ an algebraically closed field of characteristic. Set $H=\overline{\rho(G(k))}$. There exists a finite-dimensional $K$-algebra $A$, a ring homomorphism $f: k \rightarrow A$ with Zariski-dense image, and for each $\alpha \in \Phi_{k}, a$ regular map $\psi_{\alpha}: V_{\alpha}(A) \rightarrow H$ such that $\psi_{\alpha} \circ V_{\alpha}(f)=\rho \circ X_{\alpha}$.

Proof. We take $A$ and $f: k \rightarrow A$ to be the algebraic ring and ring homomorphism constructed from the restriction $\left.\rho\right|_{G_{0}(k)}$ using [16, Theorem 3.1], as described at the beginning of this section. Recall that for each $\alpha \in \Phi_{k}$, there is a regular map $\psi_{\alpha}^{1}: A_{\alpha} \rightarrow H$, and an isomorphism $\pi_{\alpha}: A \rightarrow A_{\alpha}$ such that $\pi_{\alpha} \circ f=f_{\alpha}$ and $\left.\psi_{\alpha}^{1} \circ V_{\alpha}(f)\right|_{k}=\left.\rho \circ X_{\alpha}\right|_{k}$.

First, we note that by Lemma 3.4, it suffices to construct $\psi_{\alpha}: V_{\alpha}(A) \rightarrow H$ satisfying $\psi_{\alpha} \circ V_{\alpha}(f)=$ $\rho \circ X_{\alpha}$ for a single root of each length. If $\alpha$ is a long root, then, since the corresponding root space is 1-dimensional, we can simply set $\psi_{\alpha}=\psi_{\alpha}^{1}$.

Now let us consider the short root $\alpha=\alpha_{1}-\alpha_{3}$. Then, taking $\beta=-2 \alpha_{3}$, Lemma 3.2 yields an isomorphism of algebraic varieties $\pi: B_{\alpha} \rightarrow A_{\beta}$ such that $\pi^{-1} \circ f_{\beta}=g_{\alpha}$. Define

$$
\psi_{\alpha}^{2}: A \rightarrow H, \quad \psi_{\alpha}^{2}=\iota_{B} \circ \pi^{-1} \circ \pi_{\beta},
$$

where $\pi_{\beta}: A \rightarrow A_{\beta}$ is the previously fixed isomorphism satisfying $f_{\beta}=\pi_{\beta} \circ f$, and $\iota_{B}: B_{\alpha} \hookrightarrow H$ is the natural inclusion. Using the identification $V_{\alpha}(A)=A_{L} \simeq A^{2}, v=v_{1}+v_{2} \sqrt{d} \mapsto\left(v_{1}, v_{2}\right)$, we define

$$
\psi_{\alpha}: V_{\alpha}(A) \rightarrow H, \quad v=\left(v_{1}, v_{2}\right) \mapsto \psi_{\alpha}^{1}\left(v_{1}\right) \cdot \psi_{\alpha}^{2}\left(v_{2}\right) .
$$


Note that for any $u \in k$, we have

$$
\left(\psi_{\alpha}^{2} \circ f\right)(u)=\left(\iota_{B} \circ \pi^{-1} \circ \pi_{\beta} \circ f\right)(u)=\left(\iota_{B} \circ \pi^{-1} \circ f_{\beta}\right)(u)=\left(\iota_{B} \circ g_{\alpha}\right)(u)=\left(\rho \circ X_{\alpha}\right)(u \sqrt{d})
$$

Hence, for $v=\left(v_{1}, v_{2}\right) \in V_{\alpha}(k)=L=k \oplus k \sqrt{d}$, we have

$$
\begin{aligned}
\left(\psi_{\alpha} \circ V_{\alpha}(f)\right)(v) & =\psi_{\alpha}\left(f\left(v_{1}\right), f\left(v_{2}\right)\right)=\left(\psi_{\alpha}^{1} \circ f\left(v_{1}\right)\right) \cdot\left(\psi_{\alpha}^{2} \circ f\left(v_{2}\right)\right)= \\
& =\left(\rho \circ X_{\alpha}\left(v_{1}\right)\right) \cdot\left(\rho \circ X_{\alpha}\left(v_{1} \sqrt{d}\right)\right)= \\
& =\rho \circ X_{\alpha}\left(v_{1}+v_{2} \sqrt{d}\right)=\left(\rho \circ X_{\alpha}\right)(v)
\end{aligned}
$$

Thus, $\psi_{\alpha} \circ V_{\alpha}(f)=\rho \circ X_{\alpha}$, as needed.

To conclude this section, we now show the representation $\rho$ can be lifted to a representation $\widetilde{\sigma}$ of the Steinberg group $\widetilde{G}(A)$. The precise statement is as follows.

Proposition 3.6. Let $\rho: G(k) \rightarrow \mathrm{GL}_{m}(K)$ be an abstract representation, with $K$ an algebraically closed field of characteristic 0. Set $H=\overline{\rho(G(k))}$, and let $A, f, \psi_{\alpha}$ be as in Proposition [3.5. Let $\widetilde{F}: \widetilde{G}(k) \rightarrow \widetilde{G}(A)$ be the homomorphism induced by $f$ and $\pi_{k}: \widetilde{G}(k) \rightarrow G(k)$ be the natural map. Then there exists a group homomorphism $\widetilde{\sigma}: \widetilde{G}(A) \rightarrow H$ such that $\widetilde{\sigma} \circ \widetilde{F}=\rho \circ \pi_{k}$ and $\widetilde{\sigma} \circ \widetilde{X}_{\alpha}=\psi_{\alpha}$ for all $\alpha \in \Phi_{k}$.

Proof. In order for the relation $\widetilde{\sigma} \circ \widetilde{X}_{\alpha}=\psi_{\alpha}$ to hold, we must define $\widetilde{\sigma}$ on the generators of $\widetilde{G}(A)$ by

$$
\widetilde{\sigma}\left(\widetilde{X}_{\alpha}(v)\right):=\psi_{\alpha}(v)
$$

To show that $\widetilde{\sigma}$ is well defined, we need to verify that the relations (R1) and (R2) hold, replacing $\widetilde{X}_{\alpha}$ with $\psi_{\alpha}$. For this, we imitate the proof of [16, Proposition 4.2], starting with (R1). To make the notation less burdensome, let us set $\widetilde{f}=V_{\alpha}(f)$. Let $a, b \in \widetilde{f}\left(V_{\alpha}(k)\right)$, and choose $v, w \in V_{\alpha}(k)$ such that $\tilde{f}(v)=a$ and $\widetilde{f}(w)=b$. Then

$$
\begin{aligned}
\psi_{\alpha}(a) \cdot \psi_{\alpha}(b) & =\left(\psi_{\alpha} \circ \tilde{f}\right)(v) \cdot\left(\psi_{\alpha} \circ \tilde{f}\right)(w)=\left(\rho \circ X_{\alpha}\right)(v) \cdot\left(\rho \circ X_{\alpha}\right)(w)=\rho\left(X_{\alpha}(v) \cdot X_{\alpha}(w)\right)= \\
& =\left(\rho \circ X_{\alpha}\right)(v+w)=\left(\psi_{\alpha} \circ \widetilde{f}\right)(v+w)=\psi_{\alpha}(\widetilde{f}(v)+\widetilde{f}(w))=\psi_{\alpha}(a+b) .
\end{aligned}
$$

Thus, we have two regular maps $V_{\alpha}(A) \times V_{\alpha}(A) \rightarrow H$ given by

$$
(a, b) \mapsto \psi_{\alpha}(a) \cdot \psi_{\alpha}(b) \quad \text { and } \quad(a, b) \mapsto \psi_{\alpha}(a+b)
$$

that agree on the Zariski-dense subset $\widetilde{f}\left(V_{\alpha}(k)\right) \subset V_{\alpha}(A)$. So, they must coincide on all of $V_{\alpha}(A)$. This verifies (R1). By a similar calculation, for any $\alpha, \beta \in \Phi_{k}$ with $\alpha \neq \pm \beta$, we have two regular maps $V_{\alpha}(A) \times V_{\beta}(A) \rightarrow H$ given by

$$
(a, b) \mapsto\left[\psi_{\alpha}(a), \psi_{\beta}(b)\right] \quad \text { and } \quad(a, b) \mapsto \prod_{i, j>0} \psi_{i \alpha+j \beta}\left(N_{i j}^{\alpha \beta}(a, b)\right)
$$

that agree on the Zariski-dense subset $\tilde{f}\left(V_{\alpha}(k)\right)$, and hence on all of $V_{\alpha}(A)$. Thus, (R2) holds as well. This shows that $\widetilde{\sigma}$ is well defined, and, by construction, satisfies $\widetilde{\sigma} \circ \widetilde{X}_{\alpha}=\psi_{\alpha}$ for all $\alpha \in \Phi_{k}$. Finally, for any $\alpha \in \Phi_{k}$ and $v \in V_{\alpha}(k)$, we have

$$
(\widetilde{\sigma} \circ \widetilde{F})\left(\widetilde{X}_{\alpha}(v)\right)=\widetilde{\sigma}\left(\widetilde{X}_{\alpha}(\widetilde{f}(v))\right)=\psi_{\alpha}(\widetilde{f}(v))=\left(\rho \circ X_{\alpha}\right)(v)=\left(\rho \circ \pi_{k}\right)\left(\widetilde{X}_{\alpha}(v)\right),
$$

from which it follows that $\widetilde{\sigma} \circ \widetilde{F}=\rho \circ \pi_{k}$. 


\section{Rationality AND CONCLUSION OF THE PROOF}

We retain the notations of the previous section. Namely, we let $G=\mathrm{SU}_{2 n}(L, h)$, consider an abstract representation $\rho: G(k) \rightarrow \mathrm{GL}_{m}(K)$, with $K$ an algebraically closed field of characteristic 0 , and set $H=\overline{\rho(G(k))}$. Recall that by Proposition 3.5, one can associate to $\rho$ an algebraic ring $A$ together with a ring homomorphism $f: k \rightarrow A$ with Zariski-dense image. Moreover, in Proposition 3.6, we constructed a group homomorphism $\widetilde{\sigma}: \widetilde{G}(A) \rightarrow H$ that lifts $\rho$ to a representation of the Steinberg group $\widetilde{G}(A)$. More precisely, the diagrams formed by the solid arrows below commute

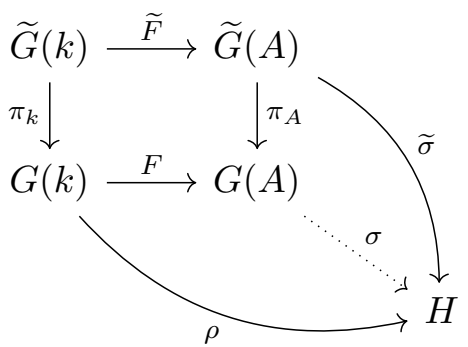

(here $F$ and $\widetilde{F}$ denote the group homomorphisms induced by $f$ ). To complete the proof of Theorem 1.2, it remains to show the existence, under the assumptions of the theorem, of a morphism of algebraic groups $\sigma: G(A) \rightarrow H$ as indicated in the diagram.

In this analysis, we are implicitly using the functor of restriction of scalars to view $G(A)$ as an algebraic $K$-group. Namely, denoting by $G_{A}$ the base change of $G$ from $k$ to $A$, the definition of the functor of restriction of scalars gives a natural isomorphism $r_{A / K}: G_{A}(A) \rightarrow R_{A / K}\left(G_{A}\right)(K)$. Since $A$ is a finite-dimensional $K$-algebra, $R_{A / K}\left(G_{A}\right)(K)$ is an affine algebraic $K$-group, and the isomorphism allows us to endow $G(A)=G_{A}(A)$ with the structure of an algebraic $K$-group.

Before addressing the algebraicity of $\sigma$, we first note the following statement, which establishes some important properties of the group $H$.

Lemma 4.1. The homomorphism $\widetilde{\sigma}: \widetilde{G}(A) \rightarrow H$ is surjective and the algebraic group $H$ is connected and perfect.

Proof. Let $\mathcal{H} \subset H$ be the (abstract) subgroup generated by the elements $\psi_{\alpha}(v)=\widetilde{\sigma} \circ \widetilde{X}_{\alpha}(v)$ for all $\alpha \in \Phi_{k}$ and $v \in V_{\alpha}(A)$, where $\psi_{\alpha}: V_{\alpha}(A) \rightarrow H$ are the regular maps introduced in Proposition 3.5. Since, by definition, the $\widetilde{X}_{\alpha}(v)$ generate $\widetilde{G}(A)$, their images generate $\widetilde{\sigma}(\widetilde{G}(A))$, so $\mathcal{H}=\widetilde{\sigma}(\widetilde{G}(A))$. Now, $A$ is connected by Remark 3.1, so $V_{\alpha}(A)$ is connected, and hence $\psi_{\alpha}\left(V_{\alpha}(A)\right)$ is connected. Thus, it follows from [2, Proposition 2.2] that $\mathcal{H}$ is Zariski-closed and connected, so $\mathcal{H} \subset H^{\circ}$. On the other hand, $\mathcal{H}$ contains $\rho(E(k))$, which is Zariski-dense in $H$. So, $\mathcal{H}$ is Zariski-dense in $H$, and since $\mathcal{H}$ is closed, we see that $\mathcal{H}=H$. This shows that $\mathcal{H}=H=H^{\circ}$. Furthermore, by Lemma 2.6, $\widetilde{G}(A)$ is equal to its commutator subgroup, so the same is true for $\mathcal{H}=\widetilde{\sigma}(\widetilde{G}(A))$.

In the remainder of the this section, we will complete the proof of Theorem 1.2 using a strategy inspired by that of $[16, \S \S 5,6]$. Namely, let $Z(H)$ be the center of $H$, set $\bar{H}=H / Z(H)$, and denote by $\nu: H \rightarrow \bar{H}$ the corresponding quotient map. We first show that $\widetilde{\sigma}$ gives rise to a group homomorphism $\bar{\sigma}: G(A) \rightarrow \bar{H}$ satisfying $\bar{\sigma} \circ \pi_{A}=\nu \circ \widetilde{\sigma}$, and verify that $\bar{\sigma}$ is in fact a morphism of algebraic groups. Then, using the assumption that the unipotent radical $U=R_{u}(H)$ is commutative (together with our standing hypothesis that char $K=0$ ), we lift $\bar{\sigma}$ to the required morphism of algebraic groups $\sigma: G(A) \rightarrow H$.

Proposition 4.2. There exists a group homomorphism $\bar{\sigma}: G(A) \rightarrow \bar{H}$ such that $\bar{\sigma} \circ \pi_{A}=\nu \circ \widetilde{\sigma}$, where $\pi_{A}: \widetilde{G}(A) \rightarrow G(A)$ is the canonical map.

Proof. First, it follows from Remarks 2.2 and 3.1 that $G(A)=E(A)$. Moreover, since $\pi_{A}$ is surjective, we have $E(A) \cong \widetilde{G}(A) / \operatorname{ker} \pi_{A}$. Now, according to Proposition 2.5, ker $\pi_{A}$ is central in $\widetilde{G}(A)$, and by Lemma 4.1, $\widetilde{\sigma}: \widetilde{G}(A) \rightarrow H$ is surjective. Consequently, we have $\widetilde{\sigma}\left(\operatorname{ker} \pi_{A}\right) \subset Z(H)$, and hence $\widetilde{\sigma}$ induces a map $\bar{\sigma}: G(A) \rightarrow \bar{H}$ on the quotients satisfying $\bar{\sigma} \circ \pi_{A}=\nu \circ \widetilde{\sigma}$. 
Next, we establish:

Proposition 4.3. The group homomorphism $\bar{\sigma}: G(A) \rightarrow \bar{H}$ from Proposition 4.2 is a morphism of algebraic groups.

Proof. By [2, Proposition 2.2], we can write $G(A)=E(A)$ as a product

$$
G(A)=\prod_{i=1}^{m} U_{\alpha_{i}}^{e_{i}}
$$

for some sequence of roots $\left\{\alpha_{1}, \ldots, \alpha_{m}\right\} \subset \Phi_{k}$, where $U_{\alpha_{i}}=X_{\alpha_{i}}\left(V_{\alpha_{i}}(A)\right)$ is the root subgroup associated with $\alpha \in \Phi_{k}$ and each $e_{i}= \pm 1$. Let $X=\prod_{i=1}^{m} A_{\alpha_{i}}$ be a product of copies of $A$ indexed by the $\alpha_{i}$, and define a regular map $s: X \rightarrow G(A)$ by

$$
s\left(a_{1}, \ldots, a_{m}\right)=X_{\alpha_{1}}\left(a_{1}\right)^{e_{1}} \cdots X_{\alpha_{m}}\left(a_{m}\right)^{e_{m}} .
$$

Let us also define a regular map $t^{\prime}: X \rightarrow H$ by

$$
t^{\prime}\left(a_{1}, \ldots, a_{m}\right)=\psi_{\alpha_{1}}\left(a_{1}\right)^{e_{1}} \cdots \psi_{\alpha_{m}}\left(a_{m}\right)^{e_{m}}
$$

where the $\psi_{\alpha_{i}}$ are the morphisms from Proposition 3.5. Set $t=\nu \circ t^{\prime}$. One easily checks that $\bar{\sigma} \circ s=t$. In particular, for any $x_{1}, x_{2} \in X$, the condition $s\left(x_{1}\right)=s\left(x_{2}\right)$ implies $t\left(x_{1}\right)=t\left(x_{2}\right)$. So, by [17, Lemma 3.10], $\bar{\sigma}$ is a rational map. Hence, there is an open subset of $G(A)$ on which $\bar{\sigma}$ is regular. Then, applying [17, Lemma 3.12], we conclude that $\bar{\sigma}$ is a morphism of algebraic groups.

To conclude the argument, we will show that $\bar{\sigma}$ can be lifted to a morphism $\sigma: G(A) \rightarrow H$. For this, we first discuss several preliminary statements, which are analogues in the present setting of results established in [16, $\S \S 5,6]$.

Let $A$ be the algebraic ring associated with the representation $\rho$, and let $J \subset A$ be its Jacobson radical. As we already noted, $A$ is a finite-dimensional $K$-algebra; in particular, $A$ is artinian, and hence $J^{d}=\{0\}$ for some $d \geq 1$ (see [1, Proposition 8.4]). Moreover, by the Wedderburn-Malcev Theorem (see [14, Theorem 11.6]), there exists a semisimple subalgebra $\bar{A} \subset A$ such that $A=\bar{A} \oplus J$ as $K$-vector spaces and $\bar{A} \cong A / J$ as $K$-algebras. We note that [16, Proposition 2.20] implies that $\bar{A} \simeq K \times \cdots \times K(r$ copies $)$. Since $G=\mathrm{SU}_{2 n}(L, h)$ is $K$-isomorphic to $\mathrm{SL}_{2 n}$, it follows that $G(\bar{A})$ is a connected, simply connected, semisimple algebraic group. For the next statement, we consider the canonical homomorphism $A \rightarrow A / J$ and set

$$
G(A, J)=\operatorname{ker}(G(A) \rightarrow G(A / J))
$$

to be the corresponding congruence subgroup. We then have the following.

\section{Lemma 4.4.}

(1) The congruence subgroup $G(A, J)$ is nilpotent.

(2) We have a Levi decomposition $G(A)=G(A, J) \rtimes G(\bar{A})$.

Proof. (1) Fixing an embedding $G(A) \hookrightarrow \mathrm{GL}_{2 n}\left(A_{L}\right)$, it is straightforward to show that

$$
\left[G\left(A, J^{a}\right), G\left(A, J^{b}\right)\right] \subset G\left(A, J^{a+b}\right)
$$

for any $a, b \in \mathbb{Z}_{\geq 1}$. Since $J$ is a nilpotent ideal, our claim follows.

(2) Using the fact that $G(A)$ is perfect (see Lemma 2.6), this statement is proved by the same argument as [16, Proposition 6.5].

Next, we note the following analogue of [16, Proposition 5.5] — this result is proved exactly as in [16], employing Lemma 4.1] in place of the corresponding statements in loc. cit. (let us point out that this is the first place where the assumption on the commutativity of the unipotent radical $U=R_{u}(H)$ is used). 
Lemma 4.5. Suppose $U=R_{u}(H)$ is commutative and char $K=0$. Then $Z(H) \cap U=\{e\}$. Moreover, in this case, $Z(H)$ is finite and is contained in any Levi subgroup of $H$.

We are now ready to complete the proof of Theorem 1.2 with the next result.

Theorem 4.6. Assume that $U=R_{u}(H)$ is commutative and char $K=0$. Then there exists a morphism of algebraic groups $\sigma: G(A) \rightarrow H$ making the diagram (4) commute.

Proof. (cf. [16, Proposition 6.6]) Let $G(A)=G(A, J) \rtimes G(\bar{A})$ be the Levi decomposition from Lemma 4.4, and set

$$
\bar{U}=\bar{\sigma}(G(A, J)), \quad \bar{S}=\bar{\sigma}(G(\bar{A})), \quad \text { and } \quad S=\left(\nu^{-1}(\bar{S})\right)^{\circ},
$$

where $\nu: H \rightarrow \bar{H}$ is the quotient map. Then $\bar{H}=\bar{U} \rtimes \bar{S}$ and $H=U \rtimes S$ are also Levi decompositions. By Lemma 4.5, we have $Z(H) \subset S$. Consequently, $\bar{S}=S / Z(H)$ and the restriction $\left.\nu\right|_{U}: U \rightarrow \bar{U}$ is an isomorphism.

Now, since the quotient map $\nu: H \rightarrow \bar{H}$ is a central isogeny, and, as we observed above, $G(\bar{A})$ is simply connected, it follows from [4, Proposition 2.24(i)] that there exists a morphism of algebraic groups $\sigma_{S}: G(\bar{A}) \rightarrow S$ such that $\left.\nu\right|_{S} \circ \sigma_{S}=\left.\bar{\sigma}\right|_{G(\bar{A})}$. Define $\sigma_{U}=\left.\nu\right|_{U} ^{-1} \circ\left(\left.\bar{\sigma}\right|_{G(A, J)}\right)$. Then

$$
\sigma=\left(\sigma_{U}, \sigma_{S}\right): G(A) \rightarrow H
$$

is a morphism of algebraic groups such that $\nu \circ \sigma=\bar{\sigma}$. It remains to show that $\sigma$ makes the diagram (41) commute. Define

$$
\chi: \widetilde{G}(A) \rightarrow H, \quad g \mapsto \widetilde{\sigma}(g)^{-1} \cdot\left(\sigma \circ \pi_{A}\right)(g) .
$$

Since $\widetilde{\sigma}$ and $\sigma \circ \pi_{A}$ are group homomorphisms, so is $\chi$. Also, by Proposition 4.2, we have $\bar{\sigma} \circ \pi_{A}=\nu \circ \widetilde{\sigma}$, which yields $\nu \circ \sigma \circ \pi_{A}=\nu \circ \widetilde{\sigma}$. Thus, the image of $\chi$ is contained in $\operatorname{ker} \nu=Z(H)$. Since $\widetilde{G}(A)$ coincides with its commutator subgroup, we conclude that $\chi$ is trivial, and hence $\sigma \circ \pi_{A}=\widetilde{\sigma}$. Finally, the equality $\sigma \circ F=\rho$ follows from the commutativity of the rest of the diagram and the surjectivity of $\pi_{k}$.

Acknowledgements. We would like to thank the anonymous referee for a careful reading of the paper and suggestions that helped to improve the exposition. The first author was partially supported by a Collaboration Grant for Mathematicians from the Simons Foundation.

\section{REFERENCES}

1. M.F. Atiyah, I.G. MacDonald, Introduction to Commutative Algebra, Westview Press, 1969.

2. A. Borel, Linear Algebraic Groups. Second Enlarged Edition, GTM 126, Springer, 1997.

3. A. Borel, J. Tits, Groupes réductifs, Publ. math. IHES 27 (1965), 55-151.

4. A. Borel, J. Tits, Complémenets à l'article: "Groupes réductifs.". Inst. Hautes Études Sci. Publ. Math. No. 41 (1972), 253-276.

5. A. Borel, J. Tits, Homomorphismes "abstraits" de groupes algébriques simples, Ann. of Math. 97 (1973), no. 3, 499-571.

6. D. Boyarchenko, I.A. Rapinchuk, On abstract representations of the groups of rational points of algebraic groups in positive characteristic, Arch. Math. (Basel) 107 (2016), no. 6, 569-580.

7. B. Conrad, O. Gabber, G. Prasad, Pseuso-reductive groups, 2nd edition, New Mathematical Monographs 26, Cambridge University Press (2015).

8. V. Deodhar, On central extensions of rational points of algebraic groups, Amer. J. Math. 100 (1978), no. 2, $303-386$.

9. J.E. Humphreys, Introduction to Lie Algebras and Representation Theory, GTM 9, Springer, 1972.

10. J.E. Humphreys, Linear Algebraic Groups, GTM 21, Springer, 1981.

11. A. Luzgarev, A. Stavrova Elementary subgroup of an isotropic reductive group is perfect, St. Petersburg Math. J. 23 (2012), no. 5, 881-890.

12. G.D. Mostow, Fully reducible subgroups of algebraic groups, Amer. J. Math. 78 (1956), $200-221$.

13. V. Petrov, A. Stavrova, Elementary subgroups of isotropic reductive groups, St. Petersburg Math. J. 20 (2009), no. 4, 625-644.

14. R.S. Pierce Associative algebras, GTM, 88. Studies in the History of Modern Science, 9. Springer-Verlag, New York-Berlin, 1982.

15. V.P. Platonov, A.S. Rapinchuk, Algebraic Groups and Number Theory, Academic Press, 1994. 
16. I.A. Rapinchuk, On linear representations of Chevalley groups over commutative rings, Proc. Lond. Math. Soc. (3) 102 (2011), no. 5, 951-983.

17. I.A. Rapinchuk, On abstract representations of the groups of rational points of algebraic groups and their deformations, Algebra Number Theory 7 (2013), no. 7, 1685-1723.

18. I.A. Rapinchuk, Abstract homomorphisms of algebraic groups and applications, in Handbook of group actions, Vol. II, 397-447, Adv. Lect. Math. (ALM) 32, Int. Press, Somerville, MA, 2015

19. I.A. Rapinchuk, On abstract homomorphisms of Chevalley groups over the coordinate rings of affine curves, Transformation Groups 24 (2019), no. 4, 1241-1259.

20. G. Seitz, Abstract homomorphisms of algebraic groups, J. London Math. Soc. (2) 56 (1997), no. 1, 104-124.

21. A. Stavrova, Homotopy invariance of non-stable $K_{1}$-functors, J. K-Theory 13 (2014), 199-248.

22. A. Stavrova, On the congruence kernel of isotropic groups over rings, Trans. Amer. Math. Soc. 373 (2020), no. 7, 4585-4626.

23. M.R. Stein, Surjective Stability in dimension 0 for $K_{2}$ and related functors, Trans. Amer. Math. Soc. 178 (1973), 165-191.

24. R. Steinberg, Lectures on Chevalley groups. Notes prepared by John Faulkner and Robert Wilson. Revised and corrected edition of the 1968 original. University Lecture Series, 66. American Mathematical Society, Providence RI (2016).

Department of Mathematics, Michigan State University, East Lansing, Mi 48824, USA

Email address: rapinchu@msu.edu

Department of Mathematics, Michigan State University, East Lansing, Mi 48824, USA

Email address: ruiterj2@msu.edu 